\title{
Practice and Exploration of Creative Thinking Training in Environmental Design Teaching
}

\author{
Zhang Jiao; Zhang Jingjing \\ Northeastern University; Tsinghua University \\ zhangjiao3002@126.com; 275252013@qq.com
}

\begin{abstract}
Based on the theoretical platform of creative thinking research, this paper starts from the relationship between creative thinking and environmental design, and uses methods like empirical research, case study and experience summary to discuss the connotation, logical relationship and thinking characteristic of creative thinking, aiming at applying creative thinking system into design methods of environmental design. Ultimately this paper will bring out a kind of creative thinking training, with rich and expanding environment design innovative thinking, that applied to environmental design teaching, so that realize the real life significance to enhance the level of environmental design creative value and guide the environmental design disciplines to build innovation training system.
\end{abstract}

Keywords-environmental design; creative thinking; analogy; symbolic metaphor

\section{INTRODUCTION}

The essence of art should be creation and personality, while the essence of design is finding and solving problems in the provisions, in which process the imagination of art creativity is needed. As the basic design theoretical system, creative thinking training, with environmental design as the starting point, focuses on the extensive, universal modern design thinking mode.

\section{A. A Summary of Creative Thinking}

\section{1) The connotation of Creative Thinking}

Creative thinking is an active way of thinking using unique method to solve problems. "The so-called creative thinking is the subject, while in practice and finding appropriate guide of problems, under the premise of solving the problem, show a sudden emergence of cognitive leap and producing new thinking [1].

\section{B. Characteristics of Creative Thinking}

1) Uniqueness: Creative thinking solves problems through a special point of thinking. a) Relevance: The extension of creative thinking can contact the object itself, as well as positive, reverse, or horizontal, vertical contact between objects and promote creativity.

b) Non-single solution: Creative thinking can lead to a variety of ideas and thinking to expand the design options, and finally implement the optimal plan.

c) Non-obviousness: Creative thinking ideas and results are not realized overnight, but often through logic and non-logical deduction and leaping to ultimately create a new artistic image.

\section{The Relationship between Creative Thinking and Environmental Design}

\section{1) Diversification of environmental design discipline}

Environmental design discipline is a new interdisciplinary develops with times. The future development of environmental design discipline is dynamic, which means a variety of origins, systems, forms, and ways. Thus, diversification is a design concept and method, an inevitable existence of contemporary environmental design. Creative thinking features constitute a unique pattern of environmental design procedures.

2) The public feature of environmental design aesthetic object

First of all, for environmental designers, there are people from different cultural backgrounds and different majors joining the field through a variety of different channels, making environmental design a multi-disciplinary and multi-content industry. Second, in terms of environmental design customers, they show multi-level social and cultural needs, so environmental design aesthetic objects are with public feature. As is shown in figure 1, figure 2, ang figure 3, it is like what Beijing people talks: the National Grand Theater is like "egg", the Olympic Sports Center is a "Bird's Nest" and the CCTV "Bird legs", which is totally different from the idea of designer. Creative thinking is a rational and scientific way of thinking that can appropriately coordinate the public features of the two. 


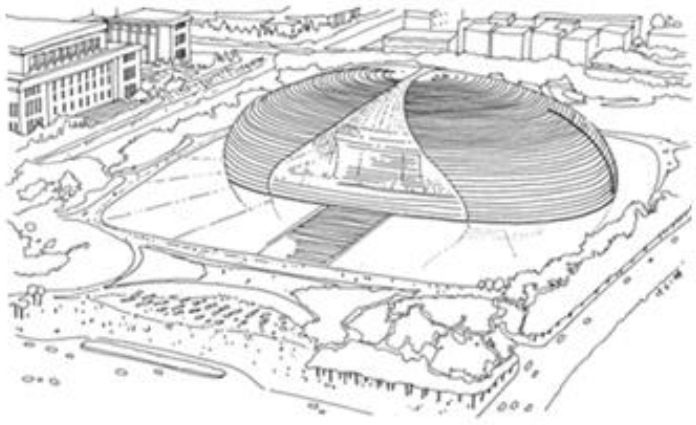

Fig 1 The National Grand Theater

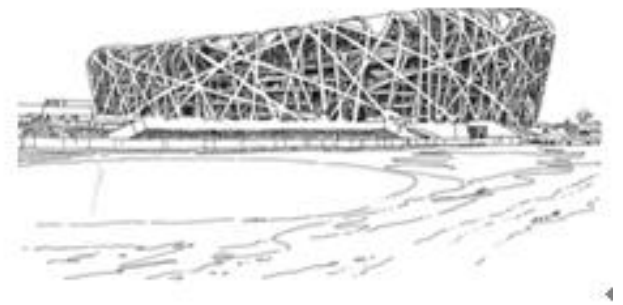

Fig 2 The Olympic Sports Center

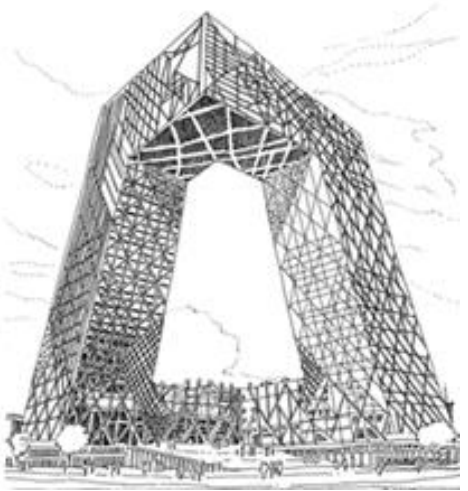

Fig 3 China Central Television Building (CCTV)

\section{The MAIN Method OF CREATIVE ThINKING TRAINING IN}

\section{ENVIRONMENTAL DESIGN}

Creative thinking is a system of research, development and effective training for people's way of thinking.

The process of environmental design is also the process of creative thinking. Under the guidance of the creative thinking mode, the designer can find the point of thinking and practice, so as to save time and design with specific aim. The efficiency of the design is reflected in the training and cultivation of creative thinking.

\section{A. Analogy}

1) The meaning of analogy

Analogy is the thinking method to compare some of the same or similar properties of the object, and find another object with these attributes. It is used to open up the horizons and explore ideas, and thus further derive Innovative design to solve the problem [2].

For example, in the figure 4, the German designer Mendelssohn designed the spiral staircase according to the shape of spiral shell, and described it as "the perfect combination of our creative thinking and natural organic creativity."
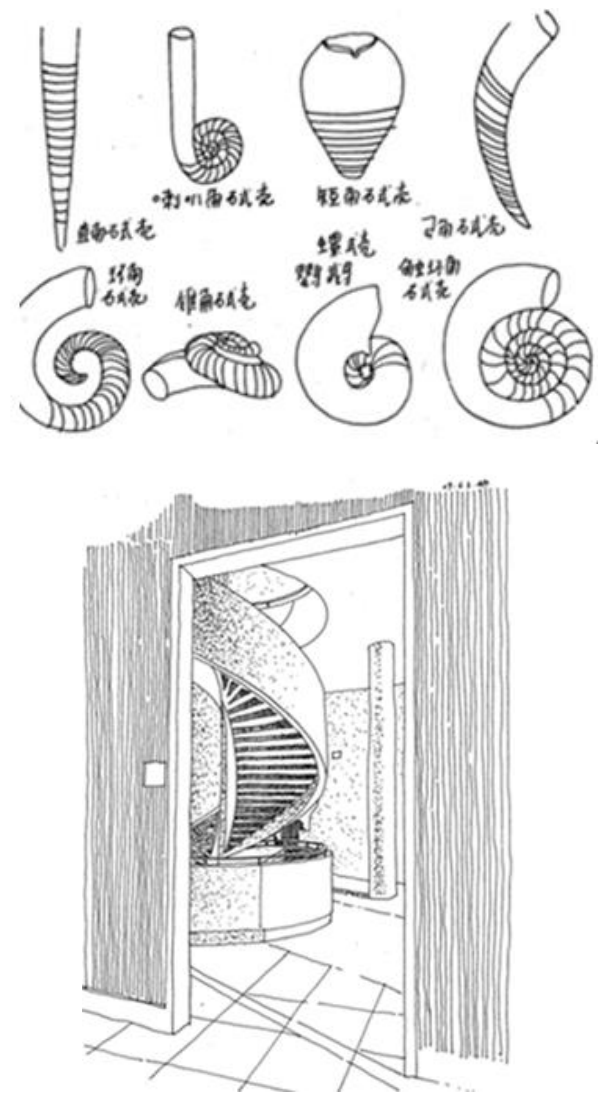

Fig 4 The spiral shell and spiral staircase

\section{B. The Classification of Analogy}

\section{1) Self-analogy}

Self-analogy is to equal the elements of the problem to be resolved with oneself, and then come up with design ideas accordingly. For example, in figure 5, the design of Long incense church, Corbusier imagined the building as himself. $\mathrm{He}$ is eager to pass the gospel of God farther, so the church's plane is designed as a horn. This is both an excellent design idea and a profound meaning.
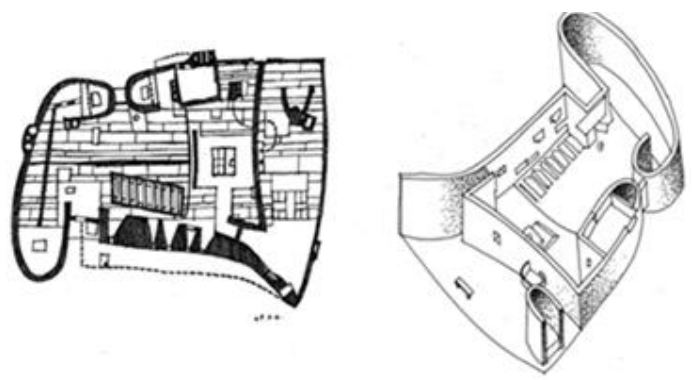

Fig 5 Longan Church Plan and Axes Diagrams 


\section{2) Imaginative analogy}

Imaginative analogy is to equal thinking things in imagination with problem to be solved to create a new way of thinking, thus stimulating creative design ideas.

Located in Chicago, Illinois, the administrative building of city's high-energy physics laboratory Fermilab's is of avant-garde style, deeply attracting physicists with the same attractive particle physics facilities inside (Figure 6). Another example is the "Dancing House" located besides the Waltava River. (Figure 7) The above two are listed as the world's top ten eccentric buildings, both typical representative using of imaginative analogy to stimulate imagination to create design.

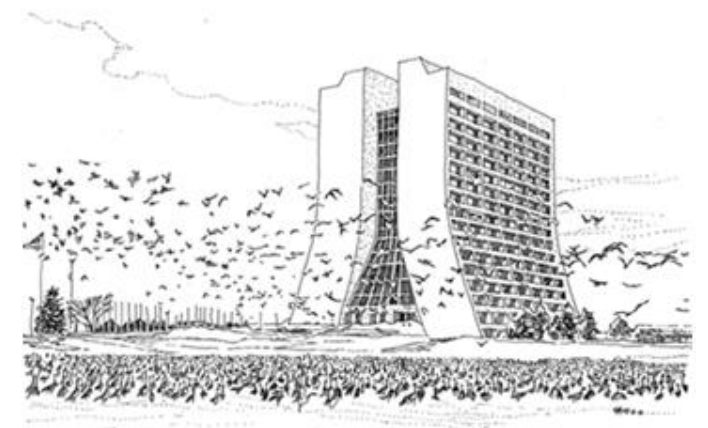

Fig 6 The world's top ten eccentric buildings - the United States Fermi National Accelerator Laboratory

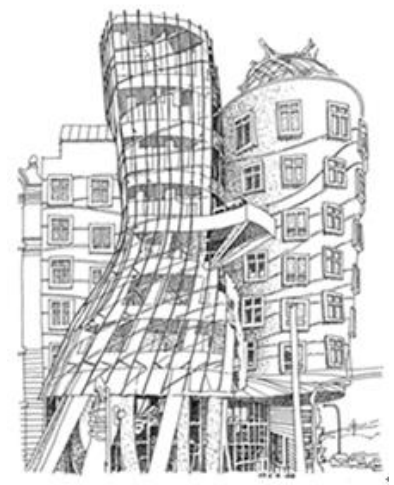

Fig 7 The world's top ten eccentric buildings - Prague "Dancing House"

\section{Symbolic metaphor}

Symbolic metaphor is a form of meaning conversion that seeing a symbol as correspond things.

1) Symbols in environmental design
a) The graphics effect

The graphical effect of symbol is to express meaning by the imitation of the image existed or resembled, borrowing the already existed meaning of the objects.

For example, as is shown in figure 8, the only geometric shape that has not been segmented by lines, circles have represent a complete, successful and unified meaning. The moon gate of Chinese traditional garden, in particular, is applauded by Chinese people for its full and harmonious shape. Shanghai Jin Mao Building also used a new interpretation of the modern process of moon door.
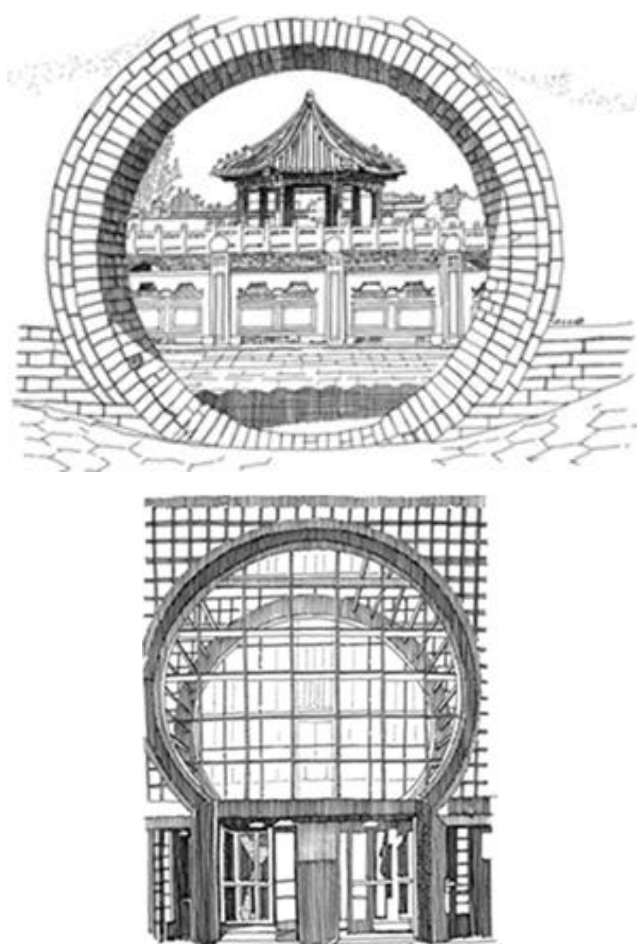

Fig 8 Chinese classical architecture of moon door and the entrance of Shanghai Jin Mao Building

\section{b) Indication effects}

The similar cognition of space, time, color, and shape in the environment may have indication effects. For example, door is an indicator of the building entrance. The guide symbol of public spaces or exhibitions and the guidance system for urban subways, stations and airports are the extensive use of indicative symbols in environmental design. Guide facilities in environmental art as is shown in figure 9. 

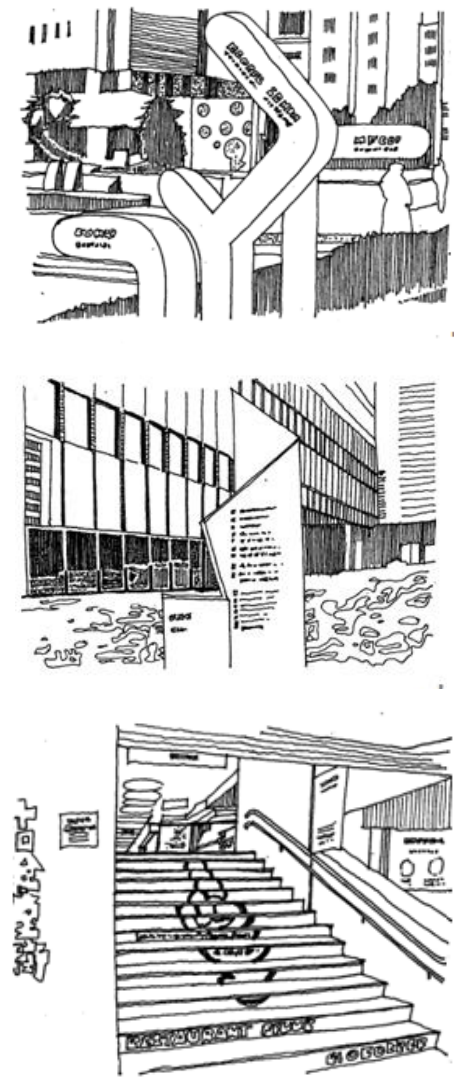

Fig 9 Guide facilities in environmental art

\section{c) Symbolic effect}

In the design of the environment, the designers have a pursuit to use symbolic approach to express a profound symbolic meaning, such as the Japanese designer Kurokawa Chancellor's design of the China-Japan Youth Exchange Center, as is shown in figure 10. The view space is designed as buddy, it is a metaphor that youth is the hope of the future; bamboo shoots form dormitory is a metaphor of the straight up character of youth; olive-like stadium is to convey the vision of peace between China and Japan; "friendly bridge" contains the long-live friendship between the two peoples.

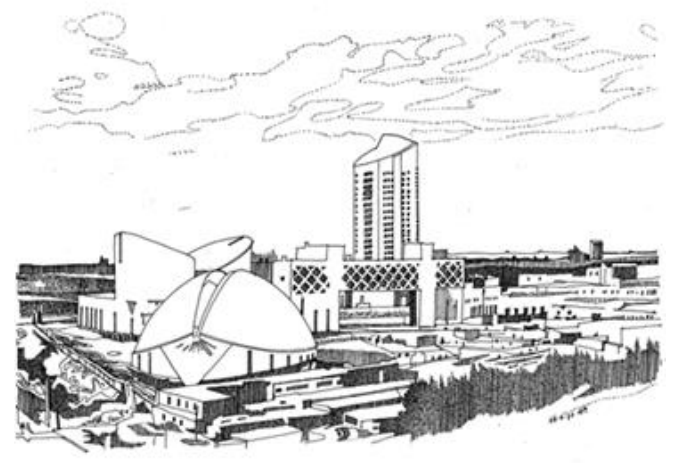

Fig 10 China-Japan Youth Exchange Center
2) Creation mechanism of symbolic metaphor - language

Language is a metaphor repository. Using language, vocabulary and concepts to state metaphor and create connection between abstract language or symbols and specific things can inspire innovative design ideas [3].

For example, in the figure 11, the graduation design works of Zhang Xin, Northeast University 2003 student, won the China Environmental Art Prize Year Award. His work, combining Chinese characters "hui"(means back) and the traditional Chinese Swirling lines decoration is designed for the Northeast University Hanqing Museum. The character "hui" in the design work is extended to meanings like "memories", "review", "back home" and so on, containing the old university president principal Zhang Xueliang's will and teachers and students' memory, perfectly further interpreting the space.
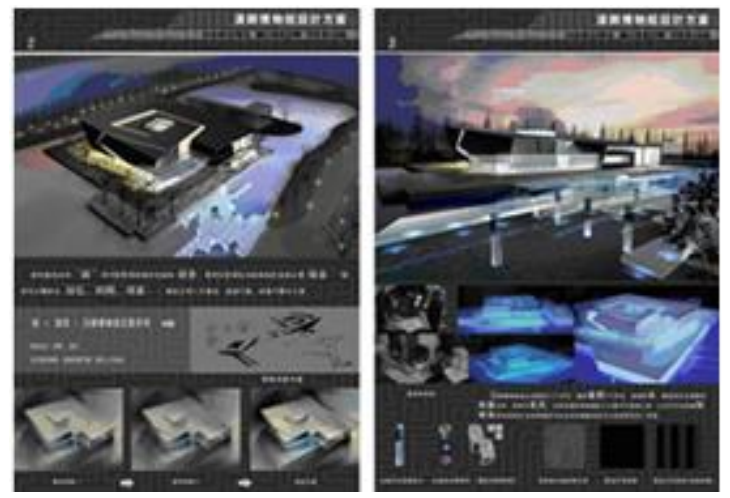

Fig 11 China Environmental Art Prize Year Award: graduation design work of Zhang Xin, Northeast University 03 - Hanqing Museum design

\section{WAYS TO IMPROVE CREATIVE THINKING ABILITY IN}

\section{ENVIRONMENTAL DESIGN}

In addition to the application of specific creative thinking methods in environmental design, the focus of methods and channels of improving imagination and cultivating creative thinking ability and other content, which is the continuation and complement of the above chapter of creative thinking training discussion, is a more potential and more essential way to improve the creative thinking ability in environmental design.

\section{A. Methods and Channels to Improve the Imagination in Environmental Design}

\section{1) Emotional Experience and Creative Imagination}

Emotional experience is a feeling with the design expectation that helps us to discover problems and ideas. In summary of the Church of Light, Ando Tadao said: "If you can arouse people's inner feelings of space and a variety of initial experiences and memories, there will be a strong resonance." [4], for example in figure 12. 


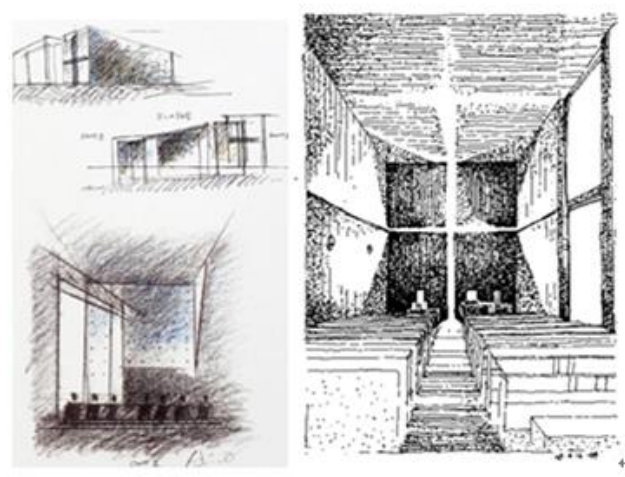

Fig 12 Ando Tadashi's Church of Light

\section{2) Image Processing and Cultural Identity}

In the environmental design, the designer design spaces to combine the perception and image of local natural conditions and historical conditions, creating a creative and new space image [5]. As is shown in figure 13, the overall planning and design of Shenzhen Vanke fifth garden is a Jiangnan Watertown-like image which simple appearance and color of white wall and black tile, combining the traditional classical elegance and postmodernism concise clever integration, cultivating a strong culture Identity.

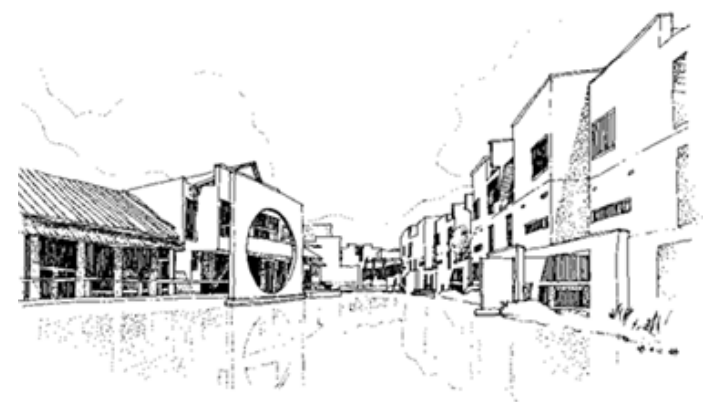

Fig 13 Shenzhen Vanke fifth park

\section{B. The focus of creative thinking ability training}

The foundation of creation lies in the potential qualities of people's mind and fitness. How to explore one's own creation potential is the most concerning question of anyone who determines to be a designer and design student [6]. To strengthen the training of creative thinking, one should proceed from the following aspects:

1) To develop imagination habits and force yourself to imagine

There's no limitation in People's imagination. We must strengthen the sense of innovation and imagine with specific purpose. Only in this way can people's initiative be mobilized, paving the way for innovation activities.

2) To expand knowledge field and add appearance reserves

Imagination is based on the existing appearance, that is, memory-based. Any imagination is based on existing knowledge. Only abundant sensitive knowledge can lead to vivid imagination.

3) To broaden the horizon and take in all-round information

In the basis of extensive reading, one should also experience the environment in real life, for the latter is three-dimensional that stored by brain in a variety of ways and thus can be mobilized through a variety of channels.

\section{4) To be curious and have sensitive emotion}

Curiosity is the starting point of creativity. One should advocate the spirit of reasonable suspicion and ask for the reason, having sensitive emotion to let imagination soar in mind.

5) To expand association and be good at fantasy

Association and fantasy are the connection and expansion of many thoughts [7]. They are often manifested by epiphany from surface to the inside and from one to another. Using this method one may be come to a higher level of seeing one and then creating more.

\section{6) To break the bondage and form creating situation}

In design, the designer should break shackles of the object concept, expressing his artistic realm freely, giving a full play of creativity.

To sum up, in the creation of environmental design, one should not only grasp the concrete way of creative thinking, adopting a reasonable way and appropriate procedures to mobilize thinking and stimulate creation of sparks, but also be cleat of how to improve creative thinking, guiding and adjusting the direction of thinking more accurately to maintain new vitality of creative design. Whenever you encounter a new design program, you can find the right path and grasp the exact direction in the process leading towards target, opening thinking tentacles in all directions. Students or designers full of thinking vitality will have a strong ideological appeal, sensitive judgment and decision-making power of environmental design.

\section{CONCLUSION}

Creative thinking training is design practice in environmental design teaching. The purpose of training is to establish a comprehensive thinking training model and to cultivate innovative ability of art and design professionals, trying to reverse and end the embarrassing situation that majority of education objects are lack of independent creative ability, realizing the transformation of modern art design study from learning to creation, from passive to active, from surface image to resonant feelings, from skill to quality.

Environmental design thinking system pursuit more the application of system approach, acknowledging design and creating from a rational and systematic point of view. In the process of design, multiple thinking training is used to create a unique creative thought. Creativity is the soul of environmental design. To explore creative thinking training methods in the field of environmental design teaching applications can help design major students or designers understand and master rules of creative thinking application, improving their ability to 
solve design problems, so as to create works full of creativity and build a bright future of modern environmental design.

\section{REFERENCES}

[1] Luo Lingling. Creative Thinking Training [M], Beijing: Capital University of Economics and Business Press, 2008. (In Chinese)

[2] Zhou Zhiyu. Thinking and Design [M], Beijing: Peking University Press, 2007. (In Chinese)
[3] Luo Lingling. Development Course of Architectural Design Creation Ability [M], Beijing: China Construction Industry Press, 2003. (In Chinese)

[4] The Church of Light [J], World Architecture, 2001.2. (In Chinese)

[5] Han Pingyi. Analysis of imagination and creative thinking in landscape design [J], modern business, 2013.11. (In Chinese)

[6] Nyingchi. On the art design course teaching [J], modern marketing, 2012.01. (In Chinese)

[7] Zhu Ying. Analysis of metaphor phenomenon in Green design [J], eco-economy, 2015.08. (In Chinese) 